\title{
Kollektive schreiben, kollektives Schreiben
}

\author{
Zur Einführung
}

\author{
Daniel Ehrmann, Thomas Traupmann
}

\section{Absagen}

„Wir schreiben zwar nicht immer darum, weil wir einsam sind“, bemerkt Johann Georg Zimmermann ${ }_{1785}$ im dritten Teil seines bekannten Buches Ueber die Einsamkeit, „aber wir müssen doch einsam seyn[,] wenn wir schreiben wollen."1 Mitten in Zimmermanns wildem Diskursgemenge, in dem sich Versatzstücke medizinischer, psychologischer, ästhetischer und ökonomischer Debatten ausmachen lassen, kommt damit der seit dem 18. Jahrhundert von Schriftsteller*innen, Kritiker*innen und Leser*innen behauptete und bis heute dominant gebliebene Normalfall von Textproduktion zu stehen, der nur eine*n Autor*in, eine Feder und einen Text vorsieht. In Zimmermanns, einer ersten Hochphase des genieästhetischen Paradigmas entstammenden, Ausführungen darf insbesondere der Protagonist philosophischer und dichterischer Schöpfungsszenen nicht ständig „seine Türe knattern, oder seine Kinder schreyen hören“, er muss ungestört „dem Triebe seines Kopfes folgen“ können, „[m]an muß ihn alleine lassen“.2 Dichtung als Funktion des Geistes, des Enthusiasmus und der Seelenkräfte ist ein einsames Geschäft, das dort endet, wo der gesellige Umgang beginnt.

Knapp zweieinhalb Jahrhunderte später scheint sich daran substanziell kaum etwas geändert zu haben: „Good novels aren't written by committee. Good novels aren't collaborated on. Good novels are produced b[y] people who voluntarily isolate themselves, and go deep, and report to the world from the depths“, ${ }^{3}$ proklamiert Jonathan Franzen, sich an Karl Kraus als Gewährsmann abarbeitend, in seiner Dankesrede anlässlich des Frank-SchirrmacherPreises 2017. Deutlicher lässt sich eine Absage an kollektives Schreiben kaum formulieren. Die Online-Crowd soll produzieren, was sie möchte, doch ihre Existenzweise bleibt allen Internet- und Technikutopien zum Trotz einzig die

1 Johann Georg Zimmermann, Ueber die Einsamkeit, Dritter Theil, Carlsruhe: Schmieder 1785, S. 305 .

2 Ebd., S. 306.

3 http://schirrmacher-stiftung.de/wp-content/uploads/2017/10/Originalrede-JonathanFranzen.pdf (letzter Zugriff: 28.03.2019). 
einer undifferenzierten, nachrangigen Menge. Diejenigen, die „serious fiction writers" sein möchten - und das heißt: die sich gar nicht erst auf die Suche nach einer "group voice" begeben -, haben einer grundsätzlichen Verantwortung zu folgen: "to continue to try to be a person“ ${ }^{4}$ Darin liege schließlich die Hauptaufgabe der ,hohen' Literatur, so resümiert Franzen, der überdies als Bewahrer eines Konzepts quasi-religiöser Genialität auftritt, wenn er in seinem Verständnis von Autorschaft ein psalmodisches (profundes) Sprechen from the depths einfordert.

\section{Konzeptualisierungen}

Solche historischen und gegenwärtigen Normierungsversuche von Schreibszenen regen gerade aufgrund der bemerkenswerten Entschiedenheit ihrer Position zum Blick über die behaupteten Grenzen an. Obgleich die starken autorschaftlichen Inszenierungen einer Produktivkraft der Einsamkeit und Versunkenheit häufig den Blick auf die Praktiken nicht nur des Schreibens, sondern eben auch des Weglegens und Wiederaufnehmens wie auch des Mitteilens und Korrigierens verdecken, so ist dennoch davon auszugehen, dass die „Erfolgsgeschichte der Autorschaft“ schon seit ihrem Beginn „von Gegenkonzepten begleitet" war. ${ }^{5}$ Wenn bereits die genannten Praktiken eine Öffnung des intimen Verhältnisses von Autor und Werk zu weiteren Akteur*innen, zu ,fremden` Händen (und Köpfen) erlauben, so hat sich durch alternative Konzepte der Textherstellung in der digitalen Netzkultur und durch die zunehmende Technisierung der Verfahren die Erosion des ohnehin prekären Singulars des Schreibens ${ }^{6}$ in den letzten Jahrzehnten noch deutlich

4 Ebd. Freilich ist dafür ein bestimmter Begriff von Person vorauszusetzen, der diskursiv, juristisch etc. überhaupt erst konsolidiert werden muss. Thomas Hobbes, dessen Schrift Leviathan als markanter und wichtiger historischer Einsatzpunkt in dieser Debatte gelten kann, bemerkt dazu unter Bezugnahme auf die lateinische Bezeichnung persona für die Maske: „A person, is he whose words or actions are considered, either as his own, or as representing the words or actions of another man, or of any other thing to whom they are attributed, whether Truly or by Fiction" (Thomas Hobbes, Leviathan, hrsg. von Crawford Brough Macpherson, Harmondsworth, New York: Penguin 1985, S. 217, Herv. im Orig.). Person zu sein impliziert eben - und hierin liegt die unfreiwillige Pointe in Franzens Postulat zugleich immer schon die Möglichkeit, hinter der Fassade oder an der Stelle eines Anderen und damit als Teil eines Kollektivs zu sprechen.

5 Michael Gamper, „Kollektive Autorschaft / kollektive Intelligenz: 1800-2000“, in: Jahrbuch der Deutschen Schillergesellschaft XLV (2001), S. 380-403, hier S. 385 .

6 Der Singular legt eine Geschlossenheit nahe, die leicht übersehen lässt, „dass Schreiben kein einheitliches Ding, sondern eine kontextsensitive Praxis ist“ (Daniel Ehrmann, „Seelenorte. 
beschleunigt. Wenn daher das Schreiben in letzter Zeit „verstärkt als Praxis in den Fokus gerückt ist, die neben den einsamen auch deutlich kooperationsoffenere Formen umfaßt ", ${ }^{7}$ so bleibt zumal aus dieser Perspektive die Geschichtsvergessenheit der software studies frappierend, deren historische Fluchtlinien insbesondere auch mit Blick auf Konzeptualisierungen des Kollektiven zu verfolgen sind (vgl. den Beitrag von MARKUs KRAJEWSKI). Betrachtet man Texte nun möglichst unbeeindruckt von den effektvollen Schöpfergesten, die sie ausgerechnet seit der „Formierungsphase der Netzwerkgesellschaft" ${ }^{\text {" }}$ im 18. Jahrhundert beinahe ausnahmslos begleiten, so erscheinen sie als deutlich komplexere Zusammenhänge. Wenn Texte also nicht mehr den Durchschlag autorschaftlicher Individualität aufs Papier darstellen, wenn sie vielmehr als Resultate sowohl von Vertextungsprozessen als auch von Vertextungshandlungen zu erachten sind, ${ }^{9}$ und wenn man sie als Hervorbringungen je unterschiedlich ausgeprägter Netzkulturen begreifen muss, dann werden sie dem dominanten Paradigma der Einsamkeit entrissen und in den Schnittpunkt diverser Kollektive versetzt. ${ }^{10}$

Zwar ist jüngst insbesondere der Begriff der Kollaboration ins Zentrum der Aufmerksamkeit gerückt, anhand dessen das Schreiben vornehmlich von der gemeinsam erbrachten Arbeit her profiliert werden kann (vgl. den Beitrag von ClaAs Morgenroth). Kollaboration meint indes, wie die Herausgeber*innen eines einschlägigen thematischen Sammelbandes festhalten, „in erster Linie Formen gemeinsamen Agierens, die ein möglichst eng aufeinander bezogenes Handeln der Einzelnen ermöglichen“, und umfasst üblicherweise solche Beziehungen, die „als hierarchiefreie oder doch zumindest -kritische

Literarische Produktion zwischen schreibenden Köpfen und denkenden Händen“, in: Susanne Knaller, Doris Pany-Habsa und Martina Scholger (Hrsg.), Schreibforschung interdisziplinär. Praxis - Prozess - Produkt, Bielefeld: transcript 2020, S. 137-155, hier S. 138).

7 Sandro Zanetti, „Einleitung“, in: ders. (Hrsg.), Schreiben als Kulturtechnik. Grundlagentexte, Berlin: Suhrkamp 2012, S. 7-34, hier S. 34.

8 Erdmut Jost, „Einführung. Das 18. Jahrhundert als Formierungsphase der Netzwerkgesellschaft", in: Erdmut Jost und Daniel Fulda (Hrsg.), Briefwechsel. Zur Netzwerkbildung in der Aufklärung, Halle/Saale: Mitteldeutscher Verlag 2012, S. 7-14, hier S. 7.

9 Für einige grundsätzliche Überlegungen zum Begriff der Vertextung vgl. Daniel Ehrmann und Thomas Traupmann, „Vertextungen. Schreiben, Schneiden, Sammeln. Workshop zur Editorik an der Universität Salzburg, 15./16. Dezember 2016“, in: editio 31 (2017), S. 254-257, sowie Daniel Ehrmann, „Vertextung. Zum relationalen Verhältnis von Autor und Werk. Mit Blick auf das Akteursnetzwerk von Luthers ,Sendbrief vom Dolmetschen“", in: Zeitschrift für deutsche Philologie 193 (2020), H. 1, S. 5-38.

Aus einem solchen Verhältnis ist immerhin auch diese Einleitung hervorgegangen. 
angelegt" sind. ${ }^{11}$ Ein Konzept des kollektiven Schreibens ist davon allerdings mindestens in zweifacher Hinsicht zu differenzieren. Zum einen wird, wie Nacim Ghanbari präzisiert, „alleinige Autorschaft“ in der Kollaboration nicht bezweifelt. ${ }^{12}$ Zeitgenössisch veranschaulicht dies etwa die Gemeinschaftspublikation Helm aus Phlox: Basierend auf einem vorangehenden Blog, der als "gemeinsame[] Materialsammlung" dient, arbeiten die fünf Autor*innen jeweils einzelne Buchkapitel aus. ${ }^{13}$ Die kollektiv signierte Vorrede kommentiert das Resultat wie folgt:

Den VerfasserInnen kommt also eine mehr oder weniger relative Autorschaft an den von ihnen betreuten Kapiteln zu, die denn auch ihrem Charakter nach ziemlich verschieden ausfallen. [...]

Wir haben dieses Buch geschrieben, und wir haben es gelesen. Nicht jede und jeder stimmt allem zu. Daraus ergibt sich, dass es sich nicht um ein Manifest oder Ähnliches handelt. Das Manifest verlangt nach einer Einheitlichkeit des Meinens, die hier nicht vorliegen kann: sie würde unseren Gegenstand planieren. ${ }^{14}$

Wenn nun Ghanbari konsequenterweise vorschlägt, die Kollaboration von kollektiver Autorschaft abzugrenzen, so ist damit über kollektives Schreiben noch nichts entschieden. Letzteres bietet nicht zuletzt deshalb die Möglichkeit, als eine Art von Hyperonym zu fungieren, das zugleich Modi der Kollaboration und der Kooperation sowie der autorschaftlichen Repräsentation umfasst, und zwar auch solche, die über den literarischen Bereich im engeren Sinne hinausführen. Das damit erweiterte Spektrum reicht bis in gegenwärtige Debatten über die akademische Publikationspraxis, die insbesondere die Naturwissenschaften und deren Vorstellung von Verfasserschaft berühren (vgl. den Beitrag

11 Nacim Ghanbari et al., „Einleitung“, in: Nacim Ghanbari et al. (Hrsg.), Kollaboration. Beiträge zur Medientheorie und Kulturgeschichte der Zusammenarbeit, Paderborn: Fink 2018, S. 1-17, hier S. 1. Zum größeren Zusammenhang vgl. Mark Terkessidis, Kollaboration, Berlin: Suhrkamp 2015.

12 Nacim Ghanbari, „Kollaboratives Schreiben im 18. Jahrhundert. Praktiken der Verbesserung und Kritik bei Gottfried August Bürger", in: Ghanbari et al. (Hrsg.), Kollaboration (Anm. 11), S. 21-37, hier S. 32.

13 Ann Cotten et al., Helm aus Phlox. Zur Theorie des schlechtesten Werkzeugs, Berlin: Merve 2011, S. 7. Dort wird in der Fußnote präzisiert: „Ann Cotten hat ausgearbeitet: Kap. 3, 10, 14; Daniel Falb: Kap. 1, 7, 17; Hendrick Jackson: Kap. 4, 12, 20; Steffen Popp: Kap. 2, 8, 16, 18; Monika Rinck: Kap. 5, 9, 15, 19. Kapitel 11 EInflusSANGST Und VATE RMORD nimmt eine gewisse Sonderstellung ein; es wurde - dem Thema angemessen - von allen gemeinsam bearbeitet."

14 Ebd., S. 8. 
von Christoph HoffmanN). ${ }^{15}$ Zum anderen ist kollektives Schreiben keinesfalls exklusiv (und erst recht nicht hierarchiefrei gedacht) mit literarischer Zusammenarbeit ${ }^{16}$ gleichzusetzen. Kollektivität und Kollegialität sind - bereits die Etymologie ${ }^{17}$ weiß um die Abgrenzung (colligere vs. *co-legare) - eben nicht schon per se zusammen zu denken. Versammlungen sind schließlich nicht bloß freie, selbstermächtigte Akte mündiger Protagonist*innen, sondern können auch erzwungene Zusammenkünfte sein und damit hierarchische Gefälle ausbilden. ${ }^{18}$ Selbst im Falle emphatisch pluraler Autorschaft werden demnach neben "kooptierter" stets auch Formen „delegierter" und "parasitärer Autorschaft" zu berücksichtigen sein. ${ }^{19}$ Außerdem gilt es zu beachten, was Hans-Jörg Rheinberger für die „literalen Anschreibetechniken“ des Labors festhält, das dann als dynamischer Verbund unterschiedlicher Dinge, Dispositive sowie technischer und menschlicher Akteure selbst zum "kollektiven Autor“ wird: Ob dort nämlich „im Modus der Konkurrenz oder der Kooperation“

15 Für die Geisteswissenschaften vgl. insbesondere den Sammelband von Stefanie Stockhorst, Marcel Lepper und Vinzenz Hoppe (Hrsg.), Symphilologie. Formen der Kooperation in den Geisteswissenschaften, Göttingen: V\&R unipress 2016. Zu nennen sind daneben etwa die großen enzyklopädischen Vorhaben des 18. Jahrhunderts oder die umfassenden Wörterbuchprojekte, für die das Deutsche Wörterbuch von Jacob und Wilhelm Grimm als repräsentativ gelten kann. Letzteres zeichnet sich dadurch aus, dass die Brüder explizit nebeneinander arbeiteten und sich folglich auf dem Titelblatt auch „als gemeinsam arbeitende Einzelforscher aus[wiesen]“ (Steffen Martus, „Literaturwissenschaftliche Kooperativität aus praxeologischer Perspektive - am Beispiel der ,Brüder Grimm“', in: ebd., S. 47-72, hier S. 51). Dazu kommt, dass sich nach dem Tod der Grimms zur Fertigstellung dieses opus magnum "immer wieder neue Formen der Kooperativität“ (ebd., S. 50) ergeben haben.

16 Vgl. dazu den Sammelband von Bodo Plachta (Hrsg.), Literarische Zusammenarbeit, Tübingen: Niemeyer 2001.

17 Vgl. die Lemmata "Kollege“ und „kollektiv“ in: Kluge. Etymologisches Wörterbuch der deutschen Sprache, bearb. von Elmar Seebold, 25., durchges. u. erw. Aufl., Berlin, Boston: de Gruyter 2011, S. 514.

18 En passant wird dieser Aspekt der unfreiwilligen oder unausweichlichen Zusammenarbeit von Nicolas Donin und Daniel Ferrer, „Auteur(s) et acteurs de la genèse“, in: Genesis 41 (2015), S. 7-26, hier S. 14, angesprochen und als „anti-collaboration“ bezeichnet.

19 Susanne Strätling, „Dieses Buch haben zwei gemacht'. Zur Praxis des Schreibens in Bündnissen“, in: Jutta Müller-Tamm, Caroline Schubert und Klaus Ulrich Werner (Hrsg.), Schreiben als Ereignis. Künste und Kulturen der Schrift, Paderborn: Fink 2018, S. 277-303, hier S. 279. 
geschrieben wird, ist zunächst einmal nachrangig. ${ }^{20}$ Zusammen-Handeln muss keineswegs implizieren, auch gemeinschaftlich zu handeln. ${ }^{21}$

Wenn sich der Begriff des Kollektivs beziehungsweise des Kollektiven nun einer solch feinen ,Segmentierung ${ }^{c}$ wie im Fall der Kollaboration (labor) oder der Kooperation (opus) entzieht, ${ }^{22}$ so speist sich - in Anlehnung an das bisher Gesagte - aus diesem Widerstand gegen eine allzu schnelle Reduzierbarkeit, mithin aus einer gewissen inhärenten Unschärfe auch sein Potenzial. Der Versuch, ein allgemeines und distinktes Konzept von Kollektivität aufzustellen, verkompliziert sich bereits angesichts der vermeintlich simplen Frage nach der Zusammensetzung von Kollektiven erheblich: Zwar erfordern sie eine bestimmte Idee respektive Fiktion von Einheitlichkeit; zugleich aber stellen sie bisweilen auch Konglomerate von „Entitäten völlig heterogener Art" dar, die nicht notwendig oder zumindest nicht zur Gänze „unter das kollektive Gebilde" subsumiert werden können. ${ }^{23}$ Überdies zeichnen sie sich durch eine gewisse Wandelbarkeit und durch ihren situativen Charakter aus - selbst dann, wenn sie unter einem vordergründig stabilen Pseudonym wie Nicolas Bourbaki agieren. ${ }^{24}$ Kollektive sind demgemäß auch auf ihre je eigene „Kopplungsenergie“ sowie ihre „spezifische Zeitlichkeit und Zeitgebundenheit“

$20 \quad$ Hans-Jörg Rheinberger, „Zettelwirtschaft“, in: Zanetti (Hrsg.), Schreiben als Kulturtechnik (Anm. 7), S. 441-452, hier S. 452. Die zweite Möglichkeit kollektiven Schreibens, die Rheinberger benennt, sind die ,Zähltechniken', die auf eine „besondere Form der Vernetzung“ von Laboratorien abzielen (ebd., S. 451).

21 Vgl. auch Urs Stäheli, „Infrastrukturen des Kollektiven: alte Medien - neue Kollektive?“, in: Zeitschrift für Medien- und Kulturforschung 3 (2012), H. 2, S. 99-116, hier S. 99.

22 Vgl. auch Claas Morgenroth, „Kollaboratives Arbeiten und kollektives Schreiben“, in: Undercurrents. Forum für linke Literaturwissenschaft 7 (2016), http://undercurrentsforum. com/index.php/undercurrents/article/view/5o/43 (letzter Zugriff: 05.04.2019), S. 2.

23 Lorenz Engell und Bernhard Siegert, „Editorial“, in: Zeitschrift für Medien- und Kulturforschung 3 (2012), H. 2, S. 5-11, hier S. 5 .

24 Darauf hat Jean Ullmo in der Diskussion anschließend an Michel Foucaults Vortrag Was ist ein Autor? hingewiesen: „Es handelt sich um ein multiples Individuum; der Name des Autors scheint sich wirklich zugunsten eines Kollektivs zu verflüchtigen, und zwar eines erneuerbaren Kollektivs, denn es sind nicht immer dieselben, die Bourbaki bilden. Gleichwohl existiert ein Autor Bourbaki, und dieser Autor Bourbaki zeigt sich in außerordentlich heftigen und ich würde sogar sagen pathetischen Diskussionen zwischen den an Bourbaki Beteiligten: vor der Veröffentlichung eines ihrer Hefte [...] finden tatsächlich nächtelange Diskussionen und Kämpfe statt, um in einem grundlegenden Gedanken Übereinstimmung zu erzielen, eine Interiorisierung“ (zit. n. Michel Foucault, „Was ist ein Autor?", in: ders., Schriften in vier Bänden. Dits et Ecrits, hrsg. von Daniel Defert und François Ewald unter Mitarb. von Jacques Lagrange, übers. von Michael Bischoff, HansDieter Gondek und Hermann Kocyba, Frankfurt/Main: Suhrkamp 2001, S. 1003-1041, hier S. 1039). 
hin zu beforschen. ${ }^{25}$ Auch die Frage, ob ihr Zusammenschluss das Resultat von Kontingenz oder Intentionalität ist, sowie die damit einhergehende nach ihrer Synchronisation wie ihren Steuerungslogiken ${ }^{26}$ werden nur fallweise zu beantworten sein. Wie sich noch zeigen wird, müssen Kollektive nicht einmal zwingend die Zeitgenossenschaft ihrer Beteiligten voraussetzen, sondern sie können auch in einer Struktur der Nachzeitigkeit existieren, ${ }^{27}$ und sie verlangen daher nach der Untersuchung ihres ausdifferenzierten Spektrums an Auftritts- und Erscheinungsformen. Der Fabrikation von Kollektiven liegt jedenfalls in der Mehrzahl der Fälle eine Gemengelage aus Diskursen, Medien und Praktiken mitsamt den ihnen inhärenten Mechanismen zugrunde.

\section{Praktiken}

Befasst man sich mit Kollektiven, so ist ganz prinzipiell nach den je involvierten Kulturtechniken zu fragen: nach den Verfahren also, durch die sie "generiert, kanalisiert und stabilisiert ${ }^{48}$ werden. Indem gerade die Konstituierung personaler Kollektive nicht nur von kollaborativem Schreiben, sondern viel häufiger noch durch den gemeinsamen Umgang mit Texten (etwa durch Lesen, Diskussion und Widerspruch oder Sanktionierung, wenn nicht gar Sakralisierung) katalysiert wird, treten Texte nicht nur als Ergebnis kollektiver Anstrengung, sondern auch als Akteure von Kollektivierung auf. Sich mit kollektiven Texten zu beschäftigen, bedeutet folglich, sie als Manifestationen spezifischer textkonstituierender oder textverändernder Handlungen sowie zugleich als Anstoß von Revisionen und Fortschreibungen zu begreifen. Wenn man kollektive Texte nach diesem Vorschlag nicht als Endpunkte einer linearen Trajektorie betrachtet, die mit einer Hand und einem Stift beginnt, um in ein Buch zu münden, sondern als Schauplätze diskontinuierlicher Verhandlungen und rekursiver Auseinandersetzungen, dann werfen sie erneut und nachdrücklich die Frage nach den Bedingungen ihres Zustandekommens auf. Was sich zunächst als Verbindung vermeintlich unauffälliger Praktiken geriert, erweist sich bei näherer Betrachtung dem Wortsinn nach

\footnotetext{
25 Engell/Siegert, „Editorial“ (Anm. 23), S. 11.

26 Vgl. Eva Horn, „Schwärme - Kollektive ohne Zentrum. Einleitung“, in: Eva Horn und Lucas Marco Gisi (Hrsg.), Schwärme - Kollektive ohne Zentrum. Eine Wissensgeschichte zwischen Leben und Information, Bielefeld: transcript 2009, S. 7-26, hier S. 10.

27 Die „Zeit der Schrift“ ermöglicht hier die „Eröffnung einer anderen Gegenwart“ (Sandro Zanetti, „Poetische Zeitgenossenschaft“, in: Variations 19 (2011), S. 39-53, hier S. 44).

28 https://www.uni-erfurt.de/projekte/kulturtechniken/veranstaltungen/tagungkulturtechniken-des-kollektiven/ (letzter Zugriff: 28.03.2019).
} 
als brisanter Verbund. Aus dieser Perspektive gerät die mittlerweile geläufige Konzentration auf Kollaboration unter Simplifizierungsverdacht, weshalb sich der vorliegende Sammelband insbesondere für das Schreiben von Kollektiven interessiert: Er gibt die vom Ergebnis ausgehende Blickrichtung auf, er will vielmehr die Kopplung des Schreibakts an das Kollektiv(e) ergründen und widmet sich daher dessen Situierung im Vertextungszusammenhang. Denn lässt man neben die abgeschlossenen Werke weitere Ergebnisse treten, dann wird sichtbar, dass die Kollektive gar nicht zwingend (literarisch) produktiv werden müssen, sondern in der gemeinsamen Tätigkeit bisweilen nichts als sich selbst hervorbringen. Die ,Freundschaftsnetze ${ }^{29}$ etwa, aus denen sich später kollaborierende Akteure rekrutieren, konstituieren sich häufig in den epistolaren Praktiken der mehrfachen Vermittlung von Lesen und Schreiben und finden mitunter in einer eigenen Kollektivpoetik ihren Niederschlag (vgl. den Beitrag von Hubert ThÜrIng). Das besonders im 18. Jahrhundert folgenreiche Konzept der Freundschaft als relativ leicht überschaubarer Form der Kollektivierung macht nicht nur deutlich, dass das selbstkonstituierende Schreiben des Kollektivs (genitivus obiectivus) vom textproduktiven Schreiben des Kollektivs (genitivus subiectivus) zu unterscheiden ist, sondern es lenkt auch den Blick auf die (potenzielle) Diskrepanz von schreibenden Händen und denkenden Köpfen. ${ }^{30}$ Die Kollektivität der Textproduktion dokumentiert sich eben nicht automatisch in unmittelbarer handschriftlicher Kollaboration. Umgekehrt spricht der Umstand, dass beim Schreiben üblicherweise „so etwas wie eine implizite Selbstarchivierung [...] des Geschriebenen “31 stattfindet, dafür, dass sich kollektive Handlungen immer wieder auch material und medial sedimentieren beziehungsweise fixieren lassen. Daran anschließend soll ausgelotet werden, wie Kollektive schreiben; wer ihnen eingeschrieben wird; wie sie er- und auch zerschrieben werden; an welchen Orten schreibende Kollektive zusammenkommen; in welchen Medien sie sich realisieren; und wer mitschreibt, ohne eigentlich teilhaben zu dürfen. Dabei greifen bisweilen auch Akteure wie der Beschreibstoff (das zu kleine Papier, der halbbrüchige Bogen, der zweckentfremdete Kalender etc.), die nie anerkannter Teil des Kollektivs waren, aktiv in den produktiven Prozess ein (vgl. den Beitrag von Cornelia OrtLieb). Schreiben ist durch all diese Implikationen in einen

29 Diese Netze werden bisweilen gezielt aufgebaut und gepflegt. Für den Jahrhundertwechsel verzeichnet Günter Oesterle, „Diabolik und Diplomatie. Freundschaftsnetzwerke in Berlin um 180o“, in: Natalie Binczek und Georg Stanitzek (Hrsg.), Strong ties / Weak ties. Freundschaftssemantik und Netzwerktheorie, Heidelberg: Winter 2010, S. 93-110, hier S. 94, einen „Re-Import von Formen der Diplomatie in die Gefühlsfreundschaft“.

30 Vgl. auch Ehrmann, „Seelenorte“ (Anm. 6).

31 Zanetti, „Einleitung“ (Anm. 7), S. 31. 
Zusammenhang von Manier, Manöver und Manipulation eingebunden, auf den Jacques Derrida aufmerksam gemacht hat. ${ }^{32}$

Eine Analyse kollektiven Schreibens wird sich an den "Umständen, Bedingungen, Verfahren und Zwängen seiner Praxis" orientieren und zugleich „die im Schreibakt sichtbar werdenden Widerstände, Funktionen und Produktionsverhältnisse" einbeziehen. ${ }^{33}$ Sie hat sich - kurz gesagt - mit der Schreibszene und der Schreib-Szene gleichermaßen zu befassen. ${ }^{34}$ Gerade wenn man Texte als kollektive Erzeugnisse begreift, werden sie noch deutlicher als Ergebnis umfassender Handlungsketten sichtbar und reflektieren die Bedingungen des Zustandekommens von Kollektivem und Kollektiven gleichermaßen. Schreiben interferiert dabei mit einer durchaus heterogenen Reihe von Verfahrensweisen: mit Schneiden und Montieren ${ }^{35}$ etwa, mit Exzerpieren, Sammeln und Kompilieren, mit Herausgeben und Edieren, mit Tilgen, Umarbeiten und Umstellen von ,fremder ${ }^{`}$ Hand, ${ }^{36}$ mit Tauschen und Zirkulieren-Lassen. Mit der schriftlichen Verfasstheit (oder allgemeiner formuliert: mit einer bearbeitbaren Form der Speicherung) eröffnet sich nun die Möglichkeit nicht nur der Trennung von der Referenz, sondern auch der Trennung von der Schreibinstanz. ${ }^{37}$ Damit ist bereits eine Grundvoraussetzung

32 Vgl. Jacques Derrida, Maschinen Papier. Das Schreibmaschinenband und andere Antworten, hrsg. von Peter Engelmann, aus dem Französischen von Markus Sedlaczek, Wien: Passagen 2006, S. 143.

33 Morgenroth, „Kollaboratives Arbeiten“ (Anm. 22), S. 5 .

34 Zu dieser Unterscheidung vgl. den einschlägigen Beitrag von Martin Stingelin, „,Schreiben؛ Einleitung“, in: ders. (Hrsg.), „Mir ekelt vor diesem tintenklecksenden Säkulum“. Schreibszenen im Zeitalter der Manuskripte, München: Fink 2004, S. 7-21.

35 Vgl. etwa auch die Aussage von Gerhard Rühm, der im Kontext der Wiener Gruppe auf die Montage hinweist als "technik, die die gemeinschaftsarbeit besonders begünstigte“ (Gerhard Rühm, „das phänomen ,wiener gruppe` im wien der fünfziger und sechziger jahre", in: ders., Aspekte einer erweiterten Poetik. Vorlesungen und Aufsätze, Berlin: Matthes \& Seitz 2008, S. 7-35, hier S. 22).

36 Zur Aufwertung von Eingriffen ,fremder' Hand im 18. Jahrhundert vgl. auch Ghanbari, „Kollaboratives Schreiben“ (Anm. 12), S. 23. Zum Lektor als notorischer Figur unsichtbarer Ko-Autorschaft vgl. Uwe Wirth, „Der Lektor als zweiter Autor“, in: Irmgard M. Wirtz, Ulrich Weber und Magnus Wieland (Hrsg.), Literatur - Verlag - Archiv, Göttingen: Wallstein 2015, S. 21-34; sowie Ines Barner, Von anderer Hand. Kollaborative Praktiken des Schreibens zwischen Autor und Lektor, Göttingen: Wallstein 2020.

37 Vgl. Jacques Derrida, „Signatur Ereignis Kontext“, in: ders., Randgänge der Philosophie, hrsg. von Peter Engelmann, aus dem Französischen von Gerhard Ahrens u.a., 2., überarb. Aufl., Wien: Passagen 1999, S. 325-351 und S. 417-419. Man wird hier zurecht auch an Bruno Latour und sein Konzept der Inskriptionen - sie umfassen das Aufschreiben, Abschreiben und Umschreiben gleichermaßen - denken, für die gilt, dass sie "mobile but also immutable, presentable, readable and combinable with one another" sind (Bruno Latour, „Visualisation and Cognition: Drawing Things Together“, in: Knowledge and 
von Zirkulation benannt, die in einen doppelten Transformationsprozess mündet: Einerseits versetzt sie Texte oder textuelle Versatzstücke in Bewegung und lässt sie verändert aus dem Zusammenspiel unterschiedlicher Instanzen und Institutionen wieder hervorgehen, was eine Re-Evaluierung ihres Zustandes als ,Text' notwendig macht; ${ }^{38}$ andererseits werden die eingebundenen Kollektive durch den Akt des Zusammen-Schreibens selbst modifiziert.

\section{Strukturen}

In welchem Maße Schreiben und Schriftlichkeit zu kollektiven Strukturen gehören, ist im Zusammenhang mit unterschiedlichen, insbesondere ,außerliterarischen' Aufschreibesystemen bereits thematisiert worden, vor allem dort, wo es im engeren Sinne um das paperwork ${ }^{39}$ geht. Das gilt etwa - wenig überraschend - für Bürokratien, insofern sie eine „komplexe Gemengelage aus Menschen, Dingen, Apparaten, Zeichenströmen und Verarbeitungsregeln“ ausbilden; ${ }^{40}$ es gilt aber auch für „halbstandardisierte Protokollformulare und Laborhandbücher"41 oder für Krankenakten als einen „kollektiven Schreibraum [...], der den Betrieb zusammenhält" ${ }^{42}$ Bereits in diesen Zusammenhängen wird deutlich, dass sich Kollektive in einem stetigen in the making befinden, ${ }^{43}$ dass sie vorrangig als relationale Gefüge funktionieren, deren Erforschung auf eine praxeologische Dimension angewiesen ist.

Kollektive sind im Moment ihrer Konstitution ein noch nicht Existierendes, das als bereits Existierendes angenommen werden muss, ${ }^{44}$ oder anders

Society. Studies in the Sociology of Culture and Present 6 (1986), S. 1-40, hier S. 7, Herv. im Orig.).

38 Schließlich können in diesem Zusammenspiel ebenso Entwürfe oder Werke produziert werden. Vgl. dazu Roland Reuß, „Schicksal der Handschrift, Schicksal der Druckschrift. Notizen zur Textgenese“, in: Text. Kritische Beiträge 5 (1999), S. 1-25, hier S. 6.

39 Latour, „Visualisation and Cognition“ (Anm. 37), S. 24.

40 Vgl. Friedrich Balke, Bernhard Siegert und Joseph Vogl, „Editorial“, in: dies. (Hrsg.), Medien der Bürokratie, Paderborn: Fink 2016, S. 5-12, hier S. 7 .

41 Rheinberger, „Zettelwirtschaft“ (Anm. 20), S. 451.

42 Christoph Hoffmann, „Schneiden und Schreiben. Das Sektionsprotokoll in der Pathologie um 1900", in: ders. (Hrsg.), Daten sichern. Schreiben und Zeichnen als Verfahren der Aufzeichnung, Zürich, Berlin: diaphanes 2008, S. 153-196, hier S. 180.

43 Vgl. auch Stäheli, „Infrastrukturen“ (Anm. 21), S. 100, der kollektives Verhalten im Sinne eines Prozesses und nicht als Aggregatform auffasst.

44 Zur Latenz des Kollektiven vgl. auch Daniel Ehrmann, „Bündnisse, die es nie gegeben hat. Lessing, Klotz und die Dynamik latenter Allianzen“, in: Franz M. Eybl, Daniel Fulda und 
gesagt: Ihre Handlungsoperationen werden zwar „im ,Kollektiv' und durch das ,Kollektiv' ausgeführt, gleichzeitig jedoch sind sie es, die das ,Kollektiv‘ überhaupt erst aufspannen und relationieren und so zusammenhalten und reproduzieren bzw. variieren. ${ }^{45}$ Dem Kollektiv wohnt damit ein veritabler double bind inne, indem es die Vorgängigkeit einer Struktur voraussetzt, die es eigentlich erst selbst etabliert. ${ }^{46}$ Demgegenüber gibt es jedoch verschiedene Möglichkeiten, in denen sich kollektive Identitäten und ihre Vertextungen infrastrukturell und juristisch, medial und generisch stabilisieren (lassen). Album, Anthologie oder scrapbook sind hier als materiale Verbünde ebenso zu nennen wie die privilegierten Gattungen kollektiver (Unter-)Zeichnung, zu denen instituierende Textsorten wie etwa die Unabhängigkeitserklärung oder das Manifest zu zählen sind. Insbesondere in letzterem widerspiegelt sich die Affinität der historischen Avantgarden zu imaginären Kollektiven wie zum kollektiven Schreiben gleichermaßen, die freilich einer Feindifferenzierung dieses Verhältnisses von Theorie und Praxis bedarf (vgl. den Beitrag von Sandro Zanetti). Durch das Aufkommen neuer Kollaborations- und Distributionsmöglichkeiten über das Internet ist auch das Online-Lexikon Wikipedia aus der Debatte nicht mehr auszusparen, das sich zudem eignet, die Veränderungen anzudeuten, welche die digitalen Netzkulturen evoziert haben: Die Möglichkeit des steten Eingriffs und der gleichsam unaufhörlichen

Johannes Süßmann (Hrsg.), Bündnisse. Politische, soziale und intellektuelle Allianzen im Jahrhundert der Aufklärung, Wien u.a.: Böhlau 2019, S. 249-273.

Engell/Siegert, „Editorial“ (Anm. 23), S. 5 .

46 Darin liegt auch die Schwierigkeit, Kollektive und Netzwerke zusammen zu denken. Zwar ist es durchaus naheliegend, an Theoreme des Netzwerks anzuknüpfen, gerade wenn es darum geht, „soziale Relationen und Abhängigkeiten in den Blick zu bekommen, die in der Literaturgeschichtsschreibung traditionell eher wenig berücksichtigt wurden“ (Hannes Fischer und Erika Thomalla, „Literaturwissenschaftliche Netzwerkforschung zum 18. Jahrhundert", in: Zeitschrift für Germanistik 26 (2016), H. 1, S. 110-117, hier S. 110). Inwiefern sie dabei mit Kollektivbildungen zusammenhängen, muss vorerst jedoch eine offene Diskussion bleiben. Anregungen dazu finden sich im Schwerpunktheft „Werke in Relationen. Netzwerktheoretische Ansätze in der Literaturwissenschaft" (hrsg. von Carlos Spoerhase und Erika Thomalla) der Zeitschrift für Germanistik 29 (2019), H. 1. Traditionellerweise werden Netzwerke zuvorderst durch ihre gegebene Konnektivität definiert (vgl. Horn, „Schwärme“ [Anm. 26], S. 16), und sie setzen im Sinne von Bauplänen voraus, dass dezidiert „sämtliche Elemente gemäß bestimmter Funktionsziele wechselseitig ergänzend, konnektiv und ausgerichtet, man kann auch sagen: formatiert und verschaltet werden“ (Hartmut Böhme, „Einführung. Netzwerke. Zur Theorie und Geschichte einer Konstruktion“, in: Jürgen Barkhoff, Hartmut Böhme und Jeanne Riou (Hrsg.), Netzwerke. Eine Kulturtechnik der Moderne, Köln u.a.: Böhlau 2004, S. 17-36, hier S. 25, Herv. d. Verf.). Sie konfigurieren demnach, so zumindest wäre eine erste Annahme zu formulieren, eine mögliche, aber nicht zwingende, Infrastruktur für Kollektive; nicht jedoch bereits das Kollektiv(e) selbst. 
Vertextung lässt Texte noch einmal stärker fluktuieren, während die Selbstbezeichnung als ,freie Enzyklopädie ein vordergründig hierarchieloses Kollektiv suggeriert, das sich realiter jedoch über ,oligarchische' Spielregeln definiert. ${ }^{47}$

Aushistorischer Perspektive haben sich außerdemZeitung undZeitschrift als privilegierte Formen kollektiven Schreibens erwiesen. ${ }^{48}$ Als avantgardistische Position prominent geworden ist etwa Sergei Tretjakows aus der Theaterpraxis abgeleitete Vorstellung von der Kollektivität der Zeitung, die mit ihrem Produktions-Artel auf ein neues, revolutionäres Bewusstsein zusteuere. ${ }^{49}$ Zeitschriften erweisen sich aber durch das Dispositiv, in das sie eingebettet sind, nicht minder auch als an literaturgeschichtlichen Konsolidierungsprozessen beteiligt, und ihre Spielregeln können in diesem Rahmen von den Beteiligten gleichermaßen produktiv aufgegriffen wie unterlaufen werden (vgl. den Beitrag von STEPHAN KAMMER). An dieser Stelle wäre zugleich der Einsatzpunkt für epochenspezifische Kollektivstile zu suchen, die überraschenderweise nur selten Probleme bereiten, sondern viel häufiger als Garanten für Authentizität aufgefasst werden, ${ }^{50}$ die aber mindestens im Falle ihrer institutionellen Rückbindungen (vgl. den Beitrag von ThомAs Assinger) zugleich auf die Gewaltimplikationen von Homogenisierungs- und Normierungsakten zu befragen sind.

Mit der Strukturierung von Kollektiven gehen schließlich jene Formierungsund Reformierungsprozesse einher, die sich im Rahmen von Schreibakten und Publikationsvorhaben zumal dann als unumgänglich zeigen, wenn die Rollen einzelner Agent*innen der Vertextung nachgezeichnet werden. Das heißt Figuren wie Schreiber*in ${ }^{51}$ und Kopist*in, Editor*in und Herausgeber*in oder Drucker*in und Setzer*in zu fokussieren (vgl. den Beitrag

47 Vgl. dazu auch Ghanbari et al., „Einleitung“ (Anm. 11), S. 1.

48 Vgl. dazu allgemein auch den kurzen Überblick von Hedwig Pompe, der zufolge die Zeitung schon ihrer formalen Gliederung nach „ihre Texte und Bilder auf Plätze in der Nachbarschaft des Vielen, Anderen [verweist]“, dabei aber dennoch gleichzeitig „Werkcharakter" erlangt (Hedwig Pompe, „Zeitung/Zeitschrift“, in: Natalie Binczek, Till Dembeck und Jörgen Schäger (Hrsg.), Handbuch Medien der Literatur, Berlin, Boston: de Gruyter 2013, S. 294-310, hier S. 296f.).

49 Vgl. Devin Fore, „Die Emergenz der sowjetischen Faktographie“, in: Deutsche Vierteljahrsschrift für Literaturwissenschaft und Geistesgeschichte 89 (2015), H. 3, S. 376-403, hier S. 386; sowie ergänzend auch Strätling, „Zur Praxis“ (Anm. 19), S. 295.

50 Vgl. Susanne Knaller, Ein Wort aus der Fremde. Geschichte und Theorie des Begriffs Authentizität, Heidelberg: Winter 2007, S. 127.

51 Zum „literary parasitism“ von (Auf-)Schreiber*innen, der einer, starken` Vorstellung von Autorschaft entgegenarbeitet, siehe auch Avital Ronell, Dictations. On Haunted Writing, Bloomington: Indiana University Press 1985, S. 65 . 
von Erika Thomalla); jene Rollen also, die an Praktiken der Beteiligung (mitschreiben, vermitteln, drucken usw.) gekoppelt sind, die zwar nicht zwingend Zugehörigkeit implizieren, aber zur Annäherung an ein Kollektiv, in manchen Fällen auch erst zu dessen Etablierung führen können. Tischszene und Diktierszene ${ }^{52}$ gestalten sich hierbei als privilegierte settings, die als Schauplätze des Kollektiven sichtbar werden (vgl. den Beitrag von ANNEgret Pelz und Christian Wimplinger). Notwendigerweise müssen dann die ,verborgenen' Praktiken, die widerständig werden können, im Auge behalten, mithin einmal mehr die ,paraskripturalen' Instanzen beziehungsweise die „skripturale[n] Parasiten“ ${ }^{53}$ bedacht werden. Für letztere gilt, wie Susanne Strätling resümiert: „Sie alle schreiben mit am Text, sie schreiben ihn bisweilen gravierend um oder auch weiter, sie korrumpieren oder sabotieren ihn, sie profitieren von ihm. ${ }^{45} \mathrm{Im}$ äußersten Extremfall wird Schreiben dabei zu einem Akt radikaler Heteronomie, der nicht von ungefähr in beinahe paradoxer Manier die ästhetische und literarische Reflexion anregt (vgl. den Beitrag von KlaUs MÜLleR-WiLle).

\section{Inszenierungen}

Mit den genannten Aspekten rücken ferner die unterschiedlichen Formen der Inszenierung von Kollektivität ins Zentrum des Interesses. Wenn etwa Goethe und Schiller in ihren Xenien individuelle autorschaftliche Positionen verunklären, provozieren sie damit zwar eine Rezeptionshaltung, die von etikettenhaften Zuschreibungen eines Textes zu einem singulären Subjekt Abstand nehmen muss; sie erzeugen indes aber auch eine figura, deren beide Köpfe auf einerEbene stehenund damit die Last der Autorfunktion gleichmäßig balancieren können. In Fällen wie diesem ist von „poetischen Praktiken des Paktierens" auszugehen, „die quer zu etablierten schematischen Alternativen von auktorialer Alleinherrschaft einerseits und Auflösung aller Autorschaftsansprüche andererseits stehen“. ${ }^{55}$ Das berühmte Bündnis mag außerdem zum Ausgangspunkt für Analysen ähnlicher Formationen werden, wobei sich

$5^{2} \mathrm{Zu}$ verschiedenen Möglichkeiten der „Mit-Autorschaft“ beim Diktieren sowie den geteilten "Souveränitätsrechte[n] mit weiteren Instanzen der Diktatszene" siehe Natalie Binczek und Cornelia Epping-Jäger, „Einleitung“, in: dies. (Hrsg.), Das Diktat. Phonographische Verfahren der Aufschreibung, Paderborn: Fink 2015, S. 7-16, hier S. 8 f. Strätling, „Zur Praxis“ (Anm. 19), S. 290, deren Auflistung „Korrektoren, Lektoren, Redakteure, Zensoren, Verleger, Kritiker, Agenten, Übersetzer usw.“ enthält.

54 Ebd.

55 Ebd., S. 303. 
die Aufgabe stellt, derartige Konstellationen aus literaturwissenschaftlicher Sicht überhaupt erst zu beschreiben und in weiterer Folge editorisch abzubilden. ${ }^{56}$ Die vielfältigen kollaborativen Formen des Schreibens wiederum, wie sie in Zeitschriftenprojekten um 18 oo zum Tragen kommen - seien es die Propyläen, das Athenäum oder die Berliner Abendblätter -, finden jeweils ganz unterschiedlichen (textuellen wie paratextuellen) Ausdruck und versetzen Herausgeber- und Autorwerk in ein notorisches Schwanken. Damit ist überdies ein fundamentaler Aspekt von Textualität berührt, der nicht zuletzt die Frage der Werkhaftigkeit betrifft. Denn es macht einen keineswegs nebensächlichen Unterschied, ob ein Textverbund als das Ergebnis einer AutorAggregation oder als das Ergebnis ,kollektiver Intelligenz' gelesen wird. ${ }^{57}$ Für den Status und die Qualität der Kategorien von Werk und Autorschaft hat es folglich durchaus Auswirkungen, wenn ein*e Autor*in weniger Organisationszentrum als vielmehr Signet eines gemeinschaftlichen Werkkomplexes wird. Selbst die mittlerweile zum Gemeinplatz erstarrte Formel vom Werk, das als ,être collectif' (Goethe) erscheint, ${ }^{58}$ verdient in diesem Kontext noch einmal neue Beachtung.

Relevant ist zudem, wer von den Beteiligten sich überhaupt als Teil des Kollektivs definiert und inszeniert. Die unwillkürliche oder beispielsweise publikationsstrategische Aktivierung vordem nur latenter Ähnlichkeitsbeziehungen etwa fordert von den Akteur*innen, sich zu dieser Gruppierung zu verhalten, sich als zugehörig oder nicht zugehörig zu positionieren. In diesem Sinne verlangen Kollektive deklarative Akte, die ihre Konstitution per se sowie die (Selbst-)Einschreibung von Beteiligten betreffen. Solche Deklarativa sind nun erstens als Aktualisierungshandlungen zu erachten, welche die grundsätzliche Zitierbarkeit eines bestimmten Rollenverhaltens voraussetzen; man wird für sie also eine allgemeine Iterierbarkeit annehmen müssen, innerhalb derer die Gefahr des Misslingens von Äußerungsakten überhaupt

$5^{6}$ Siehe Daniel Ehrmann, „Textrevision - Werkrevision. Produktion und Überarbeitung im Wechsel von Autoren, Herausgebern und Schreibern“, in: editio 30 (2016), S. 71-87.

57 Isabell Otto, „Kollektiv-Visionen. Zu den Möglichkeiten der kollektiven Intelligenz“, in: Zeitschrift für Medien- und Kulturforschung 3 (2012), H. 2, S. 185-200, hier S. 186, betont die Homogenität der Gruppierung, wenn sie zusammenfasst, dass kollektive Intelligenz zur Bezeichnung von „Tätigkeiten einer wie auch immer gestalteten Menge, die als Ganzes koordiniert ist", dient.

$5^{8}$ Zum aus dieser späten Selbstcharakterisierung gespeisten Konzept des poetischen Sprechens als Kollektivrede vgl. Kai Sina, „Wir sind viele‘. Zum Konzept dichterischer Kollektivrede bei Goethe, Ralph Waldo Emerson und Walt Whitman“, in: Comparatio 5 (2013), S. 181-203, hier S. 188; ausführlicher dazu auch Kai Sina, Kollektivpoetik. Zu einer Literatur der offenen Gesellschaft in der Moderne mit Studien zu Goethe, Emerson, Whitman und Thomas Mann, Berlin, New York: de Gruyter 2019. 
erst deren Möglichkeitsbedingung darstellt. ${ }^{59}$ Zweitens werfen die (Sprech-) Akte zugleich die Frage auf, welche „autorisierte[n] “60 Sprecher*innen überhaupt dafür einstehen. Zumal dort, wo sich Kollektive über Techniken des Dokumentarischen konstituieren, erweist sich dieser Zusammenhang durchaus als prekär: Wer spricht und darf überhaupt sprechen, wenn Fremdes dem Eigenen eingeschrieben wird? So wie Kollektive stets mit der Möglichkeit spielen, Identitäten zu supplementieren, sie von singulären zu pluralisierten Einheiten werden zu lassen, so wird im Falle dokumentarischer Praktiken das Ich zur Rede (s)eines Anderen. ${ }^{61}$ Eine poetische Praxis wie diejenige Svetlana Aleksievičs, die sich aus Zeugenschaft und der Aneignung fremder Rede gleichermaßen speist, sieht sich damit immer auch in einem Verhältnis, innerhalb dessen ,jede Fürsprache Entstellung und Entmündigung ist. ${ }^{62}$

In anderer Hinsicht verhalten sich Kollektive zu Ideen eines politisch Imaginären. ${ }^{63}$ Schon Gottfried August Bürger will 1784 seine Übersetzung der Ilias zu einem „kollaborativen Prozess"64 ausgestalten, als dessen Ergebnis ihm „die Übersetzung der ganzen Nation“65 vorschwebt. In frühromantischer Perspektive wiederum wird ein oppositionäres Verhältnis entworfen, das als Gegenüber des autoritären Autorindividuums die Vorstellung eines egalitären, ,republikanischen' Gemeinwesens konturiert, wie es sich in den berühmt gewordenen Alternativentwürfen der ,Sympoesie‘ und der ,Symphilosophie programmatisch realisieren soll. Die dadurch propagierte Form einer radikalen Kollektivierung von Literatur und Wissenschaft ist sogar darauf angelegt, die mediale Grenze des Textes zu überspringen und (zumindest imaginativ) den "Leser im Rezeptionsprozess selbst zum Schreibenden“ werden zu lassen. ${ }^{66}$ Solcherart figuriert Literatur (innerhalb des frühromantischen Kreises) als gesamtgesellschaftliches Projekt, das sich in Erweiterung des grundsätzlich unidirektionalen Textes auch der Beteiligung Außenstehender öffnet.

59 Vgl. dazu Derrida, „Signatur Ereignis Kontext“ (Anm. 37), v.a. S. 340-347.

6o John R. Searle, Ausdruck und Bedeutung. Untersuchungen zur Sprechakttheorie, übers. von Andreas Kemmerling, Frankfurt/Main: Suhrkamp 1982, S. 47.

61 Zur Denkfigur des Supplements als einer Hinzufügung in der Substitution vgl. u.a. Jacques Derrida, „Die Struktur, das Zeichen und das Spiel im Diskurs der Wissenschaften vom Menschen“, in: ders., Die Schrift und die Differenz, übers. von Rodolphe Gasché, 5. Aufl., Frankfurt/Main: Suhrkamp 1992, S. 422-442, hier v.a. S. 437.

62 Strätling, „Zur Praxis“ (Anm. 19), S. 302.

63 Vgl. Albrecht Koschorke et al., Der fiktive Staat. Konstruktionen des politischen Körpers in der Geschichte Europas, Frankfurt/Main: Fischer 2007.

64 Ghanbari, „Kollaboratives Schreiben“(Anm. 12), S. 28.

65 Gottfried August Bürger, Sämtliche Werke, hrsg. von Günter und Hiltrud Häntzschel, München, Wien: Hanser 1987, S. 678.

66 Gamper, „Kollektive Autorschaft“ (Anm. 5), S. 385. 
Es soll hierbei weniger die Frage nach der technischen Umsetzbarkeit dieser utopischen Vergemeinschaftung des Denkens im Zentrum stehen als vielmehrin Verbindung mit diesem Aspekt der Autorfunktion - die Machtstrukturen, die selbst noch der Aufforderung zur Aktivität des Zuschauers inhärent sind. ${ }^{67}$ Es scheint Teil dieses sich als Kollektivität inszenierenden autorschaftlichen Regimes zu sein, dass es sich, in Annäherung an die Konstruktion von ,Volkspoesie', selbst als beginnloses und damit herrschaftsfreies entwirft.

\section{Hierarchien}

Gewiss eines der radikalsten Konzepte hierarchieloser Kollektivität haben Gilles Deleuze und Félix Guattari in ihrer Einleitung zu Tausend Plateaus gedacht, in der es heißt:

Wir haben den ,Anti-Ödipus‘ zu zweit geschrieben. Da jeder von uns mehrere war, machte das schon eine Menge aus. Dabei haben wir alles benutzt, was uns einfiel, das Nächstliegende und das Fernste. [...] Warum wir unsere Namen behalten haben? Aus Gewohnheit, lediglich aus Gewohnheit. Um uns selbst unkenntlich zu machen. Nicht, um uns selbst zu verbergen, sondern das, was uns handeln, empfinden, denken lässt. [...] Nicht, um dabei an einen Punkt zu kommen, wo man nicht mehr ich sagt, sondern dahin, wo es völlig gleichgültig ist, ich zu sagen oder nicht. ${ }^{68}$

Neben einem solchen egalitären, rhizomatischen Verständnis des Kollektiven werden kollektive Schreibverfahren auch offensiv gegen bestehende Hierarchien in Stellung gebracht. Zu denken wäre etwa an Jean Baudrillards Kool Killers, in denen ebenfalls das Potenzial der Onymisierung der Analyse zugrundeliegt. Baudrillard liest die New Yorker Graffiti als Angriff auf die "Semiokratie“69 der urbanen Sphäre, wobei sich die Schrift der Namen als Ausweis einer „kollektiven Anonymität" gestaltet. Die Namen sind "gemacht, um verschenkt, ausgetauscht, übertragen zu werden, um sich [...] gegenseitig abzulösen“; sie entziehen sich dem Diktat des Eigentums, und sie territorialisieren den urbanen Raum neu zum „kollektiven Territorium“.70 Ein knappes Jahrhundert

67 Siehe dazu Jacques Rancière, Der emanzipierte Zuschauer, aus dem Franz. von Richard Steurer, Wien: Passagen 2009.

68 Gilles Deleuze und Félix Guattari, Rhizom, aus dem Franz. von Dagmar Berger et al., Berlin: Merve 1977, S. 5 .

69 Jean Baudrillard, Kool Killer oder Der Aufstand der Zeichen, übers. von Hans-Joachim Metzger, Berlin: Merve 1978, S. 23.

Ebd., S. 27 f. 
zuvor wiederum hat sich Cesare Lombroso mit der subversiven Komponente kollektiver Schreibverfahren befasst, die sich zu etablierten Strukturen querlegen. So verweist die Aufstellung in seinen Kerker-Palimpsesten auf einen kollektiven Schreibraum der "Schattenkommunikation “71 insbesondere in den Bänden der Gefängnisbibliotheken, mittels derer Häftlinge gegen ihre systematisch betriebene Vereinzelung - und Vereinsamung - anschreiben. In diesem Fall dient das kollektive Anschreiben jedoch einem Aufbegehren gegen Nivellierung: Schließlich bringen sich die Häftlinge darüber „als historische und kommunikative Subjekte" allererst hervor. ${ }^{72}$

Schwieriger zu beurteilen sind die Hierarchien bei Bertolt Brecht, dessen Dreigroschenproze $\beta$ als eine der „zentralen Stationen in der Theoriegeschichte

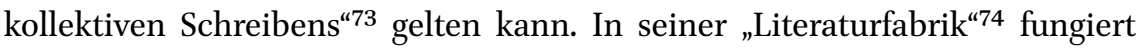
Brecht zwar zweifellos als primus inter pares, allerdings lässt er eine Reihe von Unklarheiten zurück. Diese betreffen insbesondere den Anteil seiner (freiwilligen wie unfreiwilligen) Mitarbeiter*innen an den Texten, der die Forschung ohnedies beschäftigt hat: ${ }^{75}$ In einem Fall bezahlt Brecht Personen, obwohl sie nichts zur Vertextung im engeren Sinne beigetragen haben, im nächsten marginalisiert oder tilgt er die Arbeit derjenigen, die nachweislich beteiligt waren. Auffällig ist bei Brecht ein Phänomen, mit dem sich nicht nur eine gendersensible Schreibforschung (vgl. den Beitrag von Jennifer ClARE) ganz generell zu befassen hat: dass nämlich Frauen bei kollektiven Zusammensetzungen häufig in ,minoritäre‘ Rollen innerhalb von Schreibverhältnissen gedrängt werden. So hat beispielsweise auch Conrad Ferdinand Meyers Schwester Betsy die Arbeiten des Bruders in unterschiedlichem Ausmaß mitvertextet, hat sie entworfen, korrigiert und revidiert, ist jedoch nie als Mitautorin geführt worden. ${ }^{76}$

Es scheint daher von bestimmten hierarchisch geprägten Ausgangslagen abzuhängen, ob dieselben werkkonstituierenden Textpraktiken als

71 Philipp Hubmann und Mara Persello, „Pflichtlektüren. Die Erfindung der Gefängnisbibliothek und Spuren ihrer Um-/Nutzung in Cesare Lombrosos Kerker-Palimpsesten“, in: Ulrich Leitner (Hrsg.), Corpus Intra Muros. Eine Kulturgeschichte räumlich gebildeter Körper, Bielefeld: transcript 2017, S. 85-116, hier S. 99.

72 Ebd., S. 113.

73 Morgenroth, „Kollaboratives Arbeiten“ (Anm. 22), S. 2.

74 Walter Delabar, „Brechts Factory. Zur literarischen Produktion im Zeitalter der industriellen Arbeitsteilung“, in: Plachta (Hrsg.), Literarische Zusammenarbeit (Anm. 16), S. $257-270$, hier S. 263 .

75 Vgl. zuletzt den Band von Annett Gröschner und Christian Hippe (Hrsg.), Laxheit in Fragen geistigen Eigentums. Brecht und Urheberrecht, Berlin: Verbrecher Verlag 2018.

76 Vgl. Hans Zeller, „Betsy Meyers Mitautorschaft an C. F. Meyers Werk“, in: Plachta (Hrsg.), Literarische Zusammenarbeit (Anm. 16), S. 167-195. 
Indienstnahme von Mitarbeiter*innen oder als autorschaftliche Kooperation erscheinen, und es muss daran gelegen sein, diese unterschiedlichen Ausprägungen von Machtkonstellationen sichtbar machen. Mit der Inszenierung von Kollektivität und von Kollektiven hängt eben auch zusammen, welche diskursiven Machtverhältnisse in diesen Zusammenhängen etabliert, welche Kollektive konstruiert oder forciert, welche hingegen unterschlagen werden. Werden die in Kollektiven wirksamen Machstrukturen - zumal offensiv imaginativ sistiert, fordert deren Untersuchung einen umso genaueren Blick auf die Zusammenhänge heraus. Selbst bei einem ,Strukturmodell` wie demjenigen von "Kollektive[n] ohne Zentrum“77 bleibt stets nach den „Anzeichen einer anderen Form von Gouvernementalität" ${ }^{48} \mathrm{zu}$ suchen.

Man wird nur schwer bestreiten können, „dass die Vernetzungen der Moderne von deren struktureller wie physischer Gewalt, ihren Ideologien und ihren technischen Unterdrückungs- und Kontrollapparaten nicht zu trennen sind “ ${ }^{79}$ und das gilt ebenso für Kollektive - im Extremfall bis zu total(itär)er Machtzentriertheit. Besonders drastisch zeigt sich dies etwa bei der Zwangskollektivierung von sowjetischen Autor*innen während der stalinistischen Herrschaft. ${ }^{80}$ Doch auch ganz allgemein gesprochen implizieren die Kollektivität von Schreibprozessen wie die poiesis von Kollektiven durch Schreibverfahren und Schriftlichkeit eo ipso stets schon Momente von Inklusion und Exklusion. Kollektive bilden demnach unterschiedliche integrative Qualitäten aus, bis hin zu dem Umstand, dass sie zugleich auch desintegrativ wirken, wenn - wie in manchem der genannten Beispiele - das kollektive Zustandekommen von Texten im Publikationsprozess verschleiert oder verschwiegen wird. Auch die Entscheidung zwischen Aktivität und Passivität im Prozess der Kollektivierung und Reindividualisierung liegt nur allzu häufig gar nicht bei den einzelnen Akteur*innen, sondern wird von den Machtstrukturen des Kollektivs vorgegeben. Seinesgleichen sucht in dieser Hinsicht Karl Kraus. Für den kollektiven (Text-)Verbund der Fackel und der Letzten Tage der Menschheit werden aus dem zirkulierenden Presse- und Reklamematerial sprechende Figuren herausgelöst beziehungsweise geformt, die dann vor das Kraus'sche Tribunal zitiert werden können. Dort sind sie der desavouierenden Wiederholung ${ }^{81}$ ihrer

\footnotetext{
77 Horn, „Schwärme“ (Anm. 26), S. 7.

78 Ebd., S. 8, Herv. im Orig.

79 Jürgen Barkhoff, Hartmut Böhme und Jeanne Riou, „Vorwort“, in: Barkhoff/Böhme/Riou (Hrsg.), Netzwerke (Anm. 47), S. 7-16, hier S. 10.

8o Strätling, „Zur Praxis“ (Anm. 19), S. 277.

81 Vgl. auch Cornelia Vismann, „Karl Kraus: Die Stimme des Gesetzes“, in: Deutsche Vierteljahrsschrift für Literaturwissenschaft und Geistesgeschichte 74 (2000), H. 4, S. 710-724, hier S. 720.
} 
eigenen Rede ausgesetzt und werden innerhalb der kollektiven Struktur, vernichtet'. Kraus' Herrschaftsregime ist dabei unnachgiebig wie unmissverständlich, denn für die Figuren wie für ihre historischen Vorbilder gilt, „dass sie mitsprechen müssen, aber kein Recht auf Mitsprache haben“. ${ }^{82} \mathrm{Zu}$ berücksichtigen sind auch in diesen Kontexten die diskursiven und praxeologischen Verhältnisse, die solche Text-Aggregationen möglich machen, sie im einen Fall als Zitat, im anderen als Persiflage, im wieder anderen als Plagiat erscheinen lassen. Insbesondere der letztgenannte Fall, der juristische mit literarischen Klassifikationen verschneidet, adressiert einmal mehr die Unsicherheit, dass für die Konstituierung von Kollektiven die Zustimmung aller Beteiligten überhaupt nicht erforderlich ist. Somit besteht schließlich immer auch die Option gewaltsamer Integration - das collectif involontaire - als Möglichkeit. Offen bleibt dabei jedoch, wie sich einzelne Akteur*innen dagegen zur Wehr setzen und die Machtverhältnisse wieder verschieben können. Gesetze implizieren, wie Jacques Derrida mit Blick auf Gattungsfragen festgehalten hat, eben stets auch Gegen-Gesetze in ihrem Inneren, die sie nachgerade herbeizitieren. ${ }^{83}$ Es gilt in diesem Sinn, „die Möglichkeitsbedingung und die Unmöglichkeitsbedingung einer Taxonomie ${ }^{84} \mathrm{zu}$ verhandeln, und es heißt vor allem eines nicht zu vergessen: Was Abschluss und Abschließung suggeriert, kann zugleich Schleuse sein.

82 Thomas Traupmann, „Nachschrift. Karl Kraus’ Poetik des Sekundären“, in: Jörg Paulus, Andrea Hübener und Fabian Winter (Hrsg.), Duplikat, Abschrift \& Kopie. Kulturtechniken der Vervielfältigung, Wien u.a.: Böhlau 2020, S. 261-278, hier S. 276 .

83 Vgl. Jacques Derrida, „Das Gesetz der Gattung“, in: ders., Gestade, hrsg. von Peter Engelmann, aus dem Franz. von Monika Buchgeister und Hans-Walter Schmidt, Wien: Passagen 1994, S. 245-283, hier S. 251.

84 Ebd., S. 261. 